\title{
CARBON NANOTUBE-BASED CATHODES FOR MICROWAVE TUBES*
}

\author{
M.E. Read", W.G. Schwarz, M.J. Kremer, Physical Sciences Inc., Alexandria, VA 22312, USA \\ J.D. Lennhoff, Physical Sciences Inc., Andover, MA 01810, USA \\ D.L. Carnahan, K. Kempa, Z.F. Ren, NanoLab, Inc., Watertown, MA 02172, USA
}

\begin{abstract}
Field emission cathodes based on carbon nanotubes are potential candidates for low-cost replacement of thermionic cathodes used in microwave tubes. This paper describes the technical approach for realizing and testing prototype field emission cathodes fabricated from aligned arrays of carbon nanotubes. The emission characteristics of prototype cathodes of up to $1 \mathrm{~cm}^{2}$ area have been measured in both DC and pulse mode experiments. Turnon fields as low as $10 \mathrm{kV} / \mathrm{cm}$, field enhancement factors greater than $10^{3}$, and emission currents higher than $5 \mathrm{~mA}$ have been observed. These preliminary results indicate that high current densities on the order of 1 to $10 \mathrm{~A} / \mathrm{cm}^{2}$ at practical electric fields are achievable with optimized cathode structure and improved degassing.
\end{abstract}

\section{INTRODUCTION}

Thermionic cathodes used in high-power microwave tubes are the source of many technical difficulties due to limitations on achievable current density, sensitivity to 'poisoning' materials, high-temperature operation and associated tube design restrictions, long turn-on times, and power supply requirements. Alternative types of cathodes without these technical limitations and with lower manufacturing costs have therefore long been sought. For instance, if a replacement cathode could achieve higher current densities, present design requirements for high beam compression and resulting reductions in tube gain and efficiency could be avoided, in particular in higher-frequency microwave tubes being developed for future accelerator applications.

Field emission cathodes similar to those of the Spindttype have been developed for more than a decade [1]. The development of these gated cathodes has been pursued largely for use in flat panel displays. Although at least one such cathode of about $1 \mathrm{~mm}^{2}$ area has been used to generate a $10 \mathrm{~mA}$ beam in a traveling wave tube (TWT) [2], the cathode lifetime and the ability to scale its size to areas of 10 to $100 \mathrm{~cm}^{2}$ are of concern. Other types of cold cathodes using coated or nanostructured diamond and graphite nanopowders have been under development, but the electric fields required by all of these materials for producing useful current densities remain too high to be sustainable in microwave tubes.

Carbon nanotubes (CNTs) are perfectly graphetized, helical tubes that can be produced with diameters ranging from about 2 to $100 \mathrm{~nm}$ and lengths of several microns

\footnotetext{
*Work supported by the U.S. DOE and NASA.

"read@psicorp.com, ${ }^{\ddagger}$ schwarz@ psicorp.com
}

using different production methods [3]. Research toward the development of flat panel displays and scanning microscope probes has shown that CNTs are able to carry very high local current densities of about $10^{5} \mathrm{~A} / \mathrm{cm}^{2}$ and that large enhancements of the applied electric field of more than 1000 are possible [4]. This enables observable field emission currents at electric fields of $10 \mathrm{kV} / \mathrm{cm}$ or less, and current densities greater than $1 \mathrm{~mA} / \mathrm{cm}^{2}$ at applied fields of less than $40 \mathrm{kV} / \mathrm{cm}$. Recently, current densities as high as $4 \mathrm{~A} / \mathrm{cm}^{2}$ at an applied field of 600 $\mathrm{kV} / \mathrm{cm}$ have been reported [5], but not in a configuration suitable for microwave tubes. Because carbon nanotubes are mechanically extremely strong and have good chemical stability [6], CNT cathodes are expected to have longer lifetimes and be less susceptible to ion damage than emitters made of conventional materials.

This paper describes our technical approach toward the realization of field emission cathodes produced from carbon nanotubes for use in high-power microwave tubes. We have measured the emission characteristics of prototype cathodes fabricated from aligned CNT arrays in both DC and pulse mode experiments. Our measurement results are encouraging and establish that optimization of nanotube emitter density and uniformity, as well as vacuum processing, is critical to their performance. Cathode fabrication, experimental tests, and preliminary results are discussed in the following sections.

\section{FABRICATION AND TESTING}

Carbon nanotubes are an attractive material for field emitting cathodes due to the fact that they can be grown in the form of small, sharp spikes capable of carrying very high current densities. Straight, aligned CNTs have typical base diameters of 30 to $100 \mathrm{~nm}$, and the emitting area is some fraction of that cross section. In principle, the separation between nanotubes, and thus their surface density, can be varied over a wide range. However, packing the CNTs too closely will result in self-shielding of the electric field. Simulations of the field penetration between adjacent emitters indicate that a density on the order of $10^{5}$ per $\mathrm{mm}^{2}$ would provide nearly maximum current density.

Arrays of carbon nanotubes that are grown from a uniform catalyst typically have emitter densities higher than $10^{7}$ per $\mathrm{mm}^{2}$. Figure 1 shows an SEM picture of such an aligned nanotube array. Measurable current densities are still possible from such arrays, because the apparent non-uniformity in CNT length corresponds to an effective reduction in the average emitter density. Therefore, optimization of both spacing and uniformity in the fabrication of aligned arrays is essential, in order to 
achieve maximum current density from carbon nanotubebased field emission cathodes.

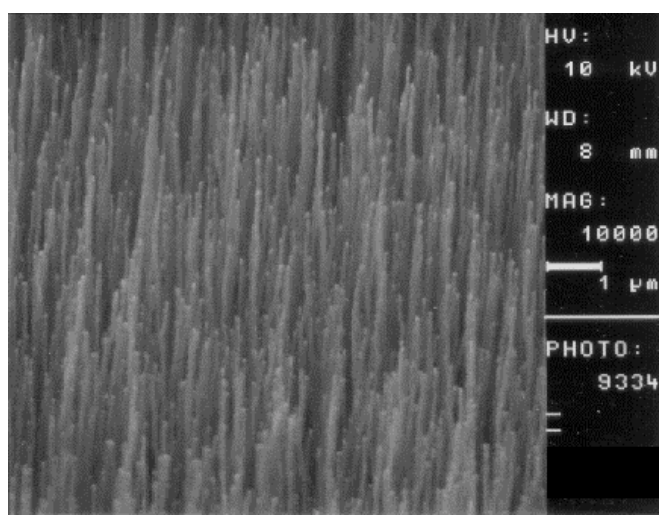

Figure 1: An aligned array of carbon nanotubes grown from a uniform catalyst.

The carbon nanotube emitters in this work have been produced using a two-step fabrication process [7]. First, a metal catalyst film is deposited onto the substrate surface. Subsequently, a plasma-enhanced CVD process is applied which initiates nanotube crystallization and promotes CNT growth into aligned arrays. Molybdenum disks of $1.27 \mathrm{~cm}$ diameter have provided the substrate material for the prototype cathodes. Typical emitter areas have ranged from about 0.4 to $1 \mathrm{~cm}^{2}$.

Most tested prototype CNT cathodes have been fabricated with emitter densities of $10^{7}$ per $\mathrm{mm}^{2}$ or higher (cf. Figure 1). To increase the spacing between emitters, initial attempts at patterning the cathode surface have been performed by depositing the catalyst through a shadow mask. The mask has consisted of a 2-D array of 8 $\mu \mathrm{m}$ wide holes with a center-to-center spacing of $12.7 \mu \mathrm{m}$. The resulting sparse CNT arrays have an average emitter density of about $10^{6}$ per $\mathrm{mm}^{2}$. Although not ideal, this lower density would be expected to improve the experimentally achievable field enhancement. However, as shown in Figure 2, the resulting carbon nanotubes are non-uniform in length and therefore would be expected to perform less than optimally.

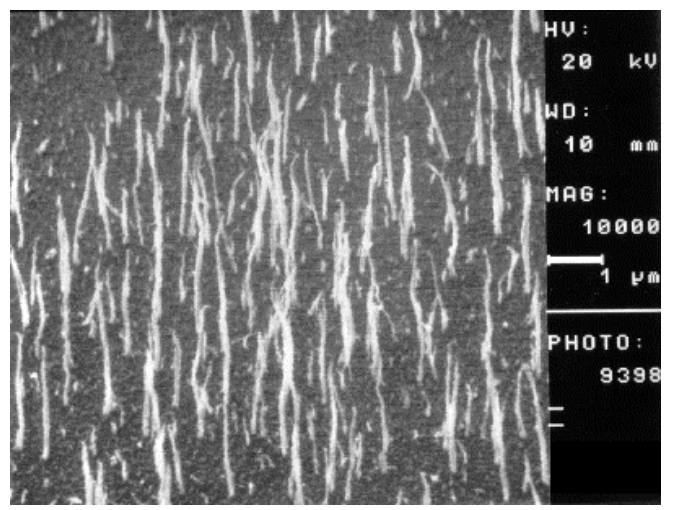

Figure 2: Carbon nanotube array grown from a patterned catalyst.

The emission characteristics of the fabricated prototype cathodes have been measured in both DC and pulse mode experiments. The test system was designed for operation with two different anode configurations. To image the beam, a hollow anode tube has been used. Designed for high current operation, the angle of the anode is appropriate for a Pierce diode. The phosphor screen installed at the end of the anode drift region has been viewed using a CCD camera. To facilitate scanning of the anode-cathode gap, an externally adjustable flat anode has been employed (replacing the hollow anode structure). The base pressure in the test chamber was better than $10^{-7}$ Torr. Measurements in DC mode have been made using a 0 to $30 \mathrm{kV}$ power supply. Pulsed tests have been performed using a 1 to $100 \mathrm{kV}$ lumped-line (soft tube) modulator with matched load. Pulse widths ranging from 1 to $10 \mu \mathrm{sec}$ with less than $2 \%$ variation during the flat portion have been applied at a pulse repetition frequency of about $1 \mathrm{~Hz}$.

\section{RESULTS AND DISCUSSION}

The results from DC testing of the fabricated prototype CNT cathodes for an anode-cathode gap of $3 \mathrm{~mm}$ are summarized in Figure 3. Emission currents higher than 5 $\mathrm{mA}$ have been reached, limited only due to heating of the uncooled anode. A measurement of the emission current as a function of the anode-cathode gap has shown that it is a function only of the applied electric field as expected. Furthermore, we have observed that careful conditioning of the cathodes at low and high currents, as well as vacuum bakeout, profoundly affects their emission characteristics, as indicated by the data in Figure 3.

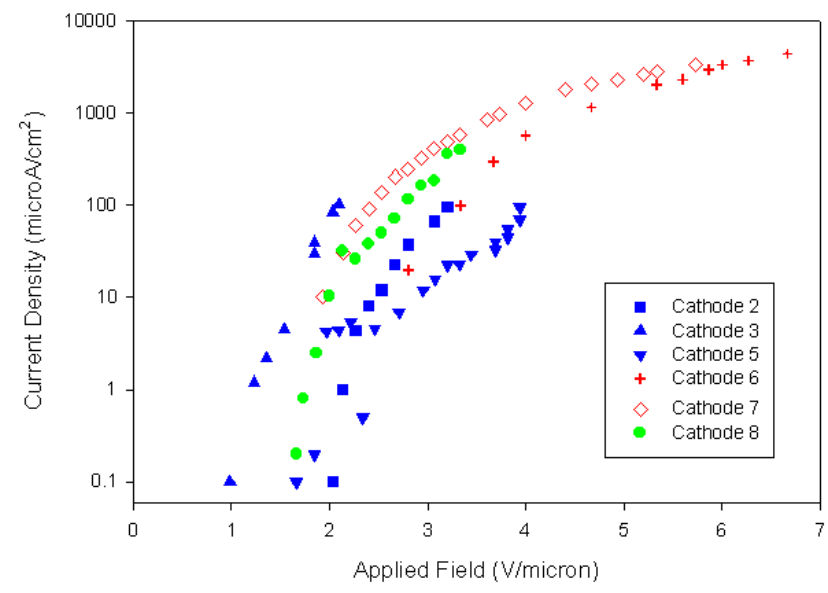

Figure 3: Test data measured in DC mode for an anodecathode gap of $3 \mathrm{~mm}$. Cathodes \#2, \#3, and \#5 were neither conditioned nor baked; cathodes \#6 and \#7 had previously been operated at the highest indicated currents, and cathode \#7 was also baked; cathode \#8 was conditioned at currents up to about $0.5 \mathrm{~mA}$ and baked.

At low currents, the emission behavior changed when the cathodes had been operated previously at currents higher than about $0.1 \mathrm{~mA}$. In this case, the turn-on field generally increased, but the emission current improved in stability and significantly higher values could be reached with good reproducibility. Bakeout of the cathodes prior to testing had a similarly beneficial effect. This behavior 
is likely due to gas adsorbed on and/or trapped inside the nanotubes. During initial operation, gas molecules are desorbed and/or expelled, thereby perhaps contributing to the emission current which subsequently drops to the actual value of the pure CNTs.

At high currents, the emission behavior changed again when the cathodes had been operated at currents higher than about $1 \mathrm{~mA}$ for several minutes. In this case, subsequent measurements showed higher emission currents at all field levels. Surprisingly, SEM images revealed that the emitter was substantially damaged. Whether this damage is caused by small discharges or by the expulsion of pockets of trapped gas from the substrate surface requires further study. Since bakeout of one cathode has resulted in some improvement, we anticipate that extensive temperature processing will eliminate this issue.

An indication of the uniformity of the emission current has been obtained from images of the phosphor screen. The result for cathode \#2 (cf. Figure 3) at $0.05 \mathrm{~mA}$ emission current is shown in Figure 4. The emission is generally found to be highly non-uniform with 'hot' spots that turn on and off at typical periods on the order of 1 second. This non-uniformity in the emission current is most probably caused by the non-uniformity in the CNT spacing and length distributions, and can be corrected by applying an improved fabrication process.

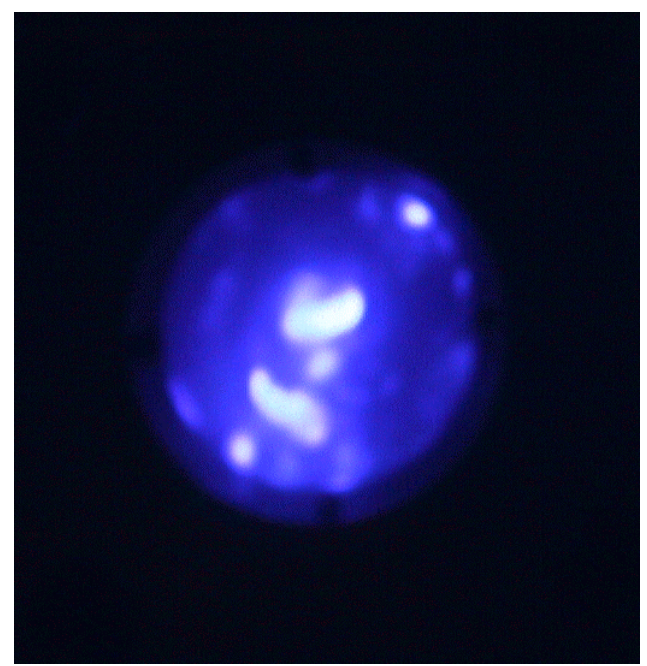

Figure 4: Image of the phosphor screen for cathode \#2 at $0.05 \mathrm{~mA}$ emission current.

During pulse testing of the prototype CNT cathodes, the electric field was limited by the formation of localized discharges between cathode and anode. The voltage at which these discharges started varied over a wide range depending on the particular cathode. For emitters that had not been conditioned at high currents above $1 \mathrm{~mA}$, this voltage limit was typically lower than $70 \%$ of the maximum voltage sustained in the DC mode. This correlation indicates that the observed behavior was caused by adsorbed and/or trapped gas. Conditioning at different currents and bakeout at high temperatures are expected to resolve this question through improved degassing.

\section{CONCLUSIONS}

Carbon nanotube-based field emitters are promising candidates for replacement of conventional cathodes in high-power microwave tubes. Our preliminary results indicate that sufficiently high current densities at practical electric fields are achievable with optimized CNT cathodes. For instance, assuming basic Fowler-Nordheim field emission behavior [8] for our prototype cathodes, the extrapolation of measured data to higher electric fields shows that current densities of about $1 \mathrm{~A} / \mathrm{cm}^{2}$ at 100 $\mathrm{kV} / \mathrm{cm}$ and about $6 \mathrm{~A} / \mathrm{cm}^{2}$ at $200 \mathrm{kV} / \mathrm{cm}$ can be achieved.

Proper conditioning and bakeout of CNT cathodes are critical to their performance, and the development of effective methods for improved degassing is continuing. Furthermore, the optimization of both spacing and uniformity in the fabrication of aligned, low-density cathode structures is essential to achieving maximum current density from carbon nanotube arrays. Toward this end, we are investigating and testing advanced lithographic methods for the production of highly uniform, large-area CNT-based field emission cathodes.

\section{REFERENCES}

[1] C.A. Spindt, C.E. Holland, and I. Brodie, IEEE Trans. Electron Devices 36 (1), 1989.

[2] R. Whaley, C.M. Armstrong, B. Ganer, P. Mukhopadhyay-Phillips, C.A. Spindt, and C.E. Holland, IEEE Int. Conf. on Plasma Science ICOPS, IEEE Conf. Abstr. 99CH36297, 1999.

[3] T.W. Ebbeson, Ann. Rev. Mater. Sci. 24, p. 235, 1994.

[4] W.A. deHeer, A. Chatelain, and D. Ugarte, Science 270, p. 1179, 1995.

[5] W. Zhu, C. Bower, O. Zhou, G. Kochanski, and S. Jin, Appl. Phys. Lett. 75 (6), p. 873, 1999.

[6] Y. Saito and S. Uemura, Carbon 38 (2), p. 169, 2000.

[7]Z.F. Ren, Z.P. Huang, J.W. Xu, J.H. Wang, P. Bush, M.P. Siegal, and P.N. Provencio, Science 282, p. 1105, 1998.

[8] R.H. Fowler and L.W. Nordheim, Proc. Royal Soc. London A 119, p. 173, 1928. 